This item was submitted to Loughborough's Research Repository by the author.

Items in Figshare are protected by copyright, with all rights reserved, unless otherwise indicated.

\title{
A novel semi-blind signal extraction approach incorporating parafac for the removal of eye-blink artifact from EEGs
}

PLEASE CITE THE PUBLISHED VERSION

\section{PUBLISHER}

(c) IEEE

\section{VERSION}

VoR (Version of Record)

\section{LICENCE}

CC BY-NC-ND 4.0

\section{REPOSITORY RECORD}

Nazarpour, K., Saeid Sanei, and Jonathon Chambers. 2019. "A Novel Semi-blind Signal Extraction Approach Incorporating Parafac for the Removal of Eye-blink Artifact from Eegs". figshare.

https://hdl.handle.net/2134/5638. 
This item was submitted to Loughborough's Institutional Repository (https://dspace.lboro.ac.uk/) by the author and is made available under the following Creative Commons Licence conditions.

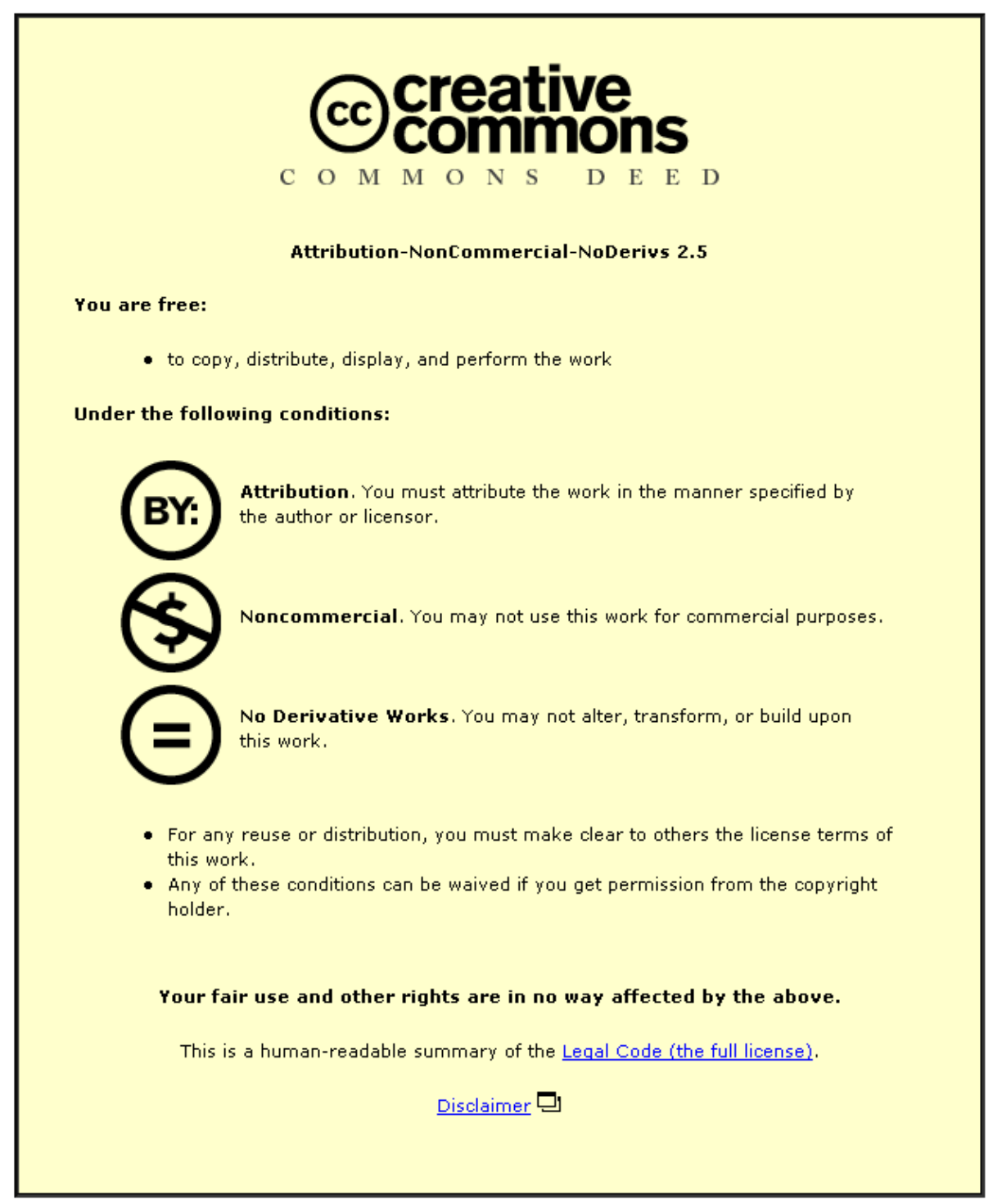

For the full text of this licence, please go to: http://creativecommons.org/licenses/by-nc-nd/2.5/ 


\title{
A NOVEL SEMI-BLIND SIGNAL EXTRACTION APPROACH INCORPORATING PARAFAC FOR THE REMOVAL OF EYE-BLINK ARTIFACT FROM EEGS
}

\author{
Kianoush Nazarpour, Saeid Sanei, and Jonathon A. Chambers \\ Centre of Digital Signal Processing, School of Engineering, Cardiff University, Cardiff CF24 3AA, UK
}

\begin{abstract}
In this paper, a novel iterative blind signal extraction (BSE) scheme for the removal of the eye-blink artifact from electroencephalogram (EEG) signals is proposed. In this method, in order to remove the artifact, the signal extraction algorithm is provided with a priori information, i.e., an estimation of the column of the mixing matrix corresponding to the eyeblink source. The a priori knowledge, namely the vector corresponding to the spatial distribution of the eye-blink factor, is identified by using the method of parallel factor analysis (PARAFAC). Hence, we call the BSE approach, semiblind signal extraction (SBSE). The results demonstrate that the proposed algorithm effectively identifies and removes the eye-blink artifact from raw EEG measurements.
\end{abstract}

Index Terms - Artifact removal, blind signal extraction, electroencephalogram, eye-blink, and parallel factor analysis.

\section{INTRODUCTION}

An electroencephalogram (EEG) is the manifestation of brain activity recorded as changes in electrical potentials at multiple locations over the scalp. An electrooculogram (EOG) generated by eye movements and/or blinks is found to be the most significant and common interfering artifact in EEG [1] since eye movements are difficult to suppress over a sustained recording course. Such an EOG is of the order of ten times larger in amplitude than average cortical signals and lasts approximately $300 \mathrm{msec}$. Due to the magnitude of the blinking artifacts and the high resistance of the skull and scalp tissues, EOG may contaminate the majority of the electrodes, even those in the occipital area. In recent years, various methods for eye-blink removal from EEGs have been proposed that are mainly based on regression methods and independent component analysis (ICA) [1, 2]. Approaches, such as trial rejection, eye fixation, EOG subtraction, and principal component analysis (PCA) [3], regression [4] and blind source separation (BSS) $[5,6]$ have been documented with varying success. Despite no quantitative comparison for any reference dataset being available, it has been shown that the regression- and BSS-based methods are most reliable [1, 4, 6] and [7].

This work is supported by The Leverhulme Trust, UK. e-mails:\{NazarpourK, SaneiS, ChambersJ\}@cf.ac.uk
Statistically nonstationary EEG signals yield information about active parts of the brain. This spatial knowledge has been efficiently exploited for localizing the sources of background EEG, using common spatial factor decomposition of the EEGs within the PCA paradigm [8]. However, the use of PCA introduces nonuniqueness due to an arbitrary choice of rotation axes. Recently, ICA has been applied to eliminate this nonuniqueness problem by imposing the statistical independence constraint which is stronger than the orthogonality condition exploited by PCA [9]. However, in conventional PCA/ICA, no other prior knowledge, such as frequency band, is exploited during the extraction process. Recently, in [10], the authors exploited a space-time-frequency (STF) model of a multi-channel EEG within the three-way (PARAFAC) [9] scheme to obtain the spatial, temporal and spectral signatures of EEGs.

In this paper, a novel hybrid BSE-PARAFAC technique for removing the EOG artifacts from multi-channel EEGs is presented. Our BSE method is based on that introduced in [11], while the spatial a priori knowledge of the mixing process obtained by PARAFAC analysis is exploited in the extraction procedure. In contrast to many BSS- and constrained BSS-based methods [1, 5] and [7], which require high computational cost, this method is simple since it extracts just the source of interest (SoI), i.e., the eye-blink source. Moreover, unlike methods presented in [5] and [6], there is no need to compute objective criteria for distinguishing between eyeblink and spurious peaks in the ongoing EEGs. Besides, unlike the regression-based methods, it does not need any reference EOG channel recordings.

This paper is organized as follows. In Section II, we present the SBSE method and briefly review the PARAFAC. The results are subsequently reported in Section III, followed by concluding remarks in Section IV.

\section{ALGORITHM DEVELOPMENT}

Assume $N$ zero-mean real mutually uncorrelated sources $\mathbf{s}(t)=$ $\left[s_{1}(t), s_{2}(t), \cdots, s_{N}(t)\right]^{T}$, where $[\cdot]^{T}$ denotes the vector transpose, are mixed by an $N \times N$ real full column rank matrix $\mathbf{A}=\left[\mathbf{a}_{1}, \mathbf{a}_{2}, \cdots, \mathbf{a}_{N}\right]$ where $\mathbf{a}_{i}$ is the $i$ th column of $\mathbf{A}$. The vector of time mixture samples $\mathbf{x}(t)=\left[x_{1}(t), x_{2}(t), \cdots, x_{N}(t)\right]^{T}$ 
is given as

$$
\mathbf{x}(t)=\mathbf{A} \mathbf{s}(t)+\mathbf{v}(t)
$$

where $\mathbf{v}(t)=\left[v_{1}(t), v_{2}(t), \cdots, v_{N}(t)\right]^{T}$ is the additive white Gaussian zero-mean noise. We assume that the sensor noise is spatially uncorrelated with the sensor data and temporally uncorrelated. Since the sources are presumed to be uncorrelated, the time lagged autocorrelation matrix $\mathbf{R}_{k}$ can be calculated as $\mathbf{R}_{k}=E\left[\mathbf{x}(t) \mathbf{x}^{T}\left(t-\tau_{k}\right)\right]$ for $k=1,2, \cdots, K$, where $K$ is the index of the maximum time lag, i.e., $\tau_{K}$ and $E[\cdot]$ denotes the statistical expectation operator.

\subsection{Semi-Blind Signal Extraction}

The vector $\mathbf{x}(t)$ in (1) is a linear combination of the columns of the mixing matrix, i.e., the $\mathbf{a}_{i} \mathrm{~s}$, weighted by the associated source and contaminated by sensor noise $\mathbf{v}(t)$. Therefore, the most straightforward way to extract the $j$ th source is to project $\mathbf{x}(t)$ onto the space in $\mathcal{R}^{N}$ orthogonal to, denoted by $\perp$, all of the columns of $\mathbf{A}$ except $\mathbf{a}_{j}$, i.e., $\left\{\mathbf{a}_{1}, \cdots, \mathbf{a}_{j-1}, \mathbf{a}_{j+1}, \cdots, \mathbf{a}_{N}\right\}$ Hence, by defining a vector $\mathbf{p} \perp\left\{\mathbf{a}_{1}, \cdots, \mathbf{a}_{j-1}, \mathbf{a}_{j+1}, \cdots, \mathbf{a}_{N}\right\}$ and $\mathbf{q} \equiv \mathbf{a}_{j}$, and adopting the notation of an oblique projector [11], we may write $y(t) \mathbf{q}=\mathbf{E}_{\mathbf{q} \mid \mathbf{p}^{\perp}} \mathbf{x}(t)$ where $y(t)$ is an estimate of one source, say $s(t)$, and $\mathbf{p}^{\perp}$ denotes the space in $\mathcal{R}^{N}$ orthogonal to $\mathbf{p}$, i.e., $\left\{\mathbf{a}_{1}, \cdots, \mathbf{a}_{j-1}, \mathbf{a}_{j+1}, \cdots, \mathbf{a}_{N}\right\}$. $\mathbf{E}_{\mathbf{q} \mid \mathbf{p}^{\perp}}=\frac{\mathbf{q p}^{T}}{\mathbf{p}^{T} \mathbf{q}}$ represents the oblique projection of $\mathbf{q}$ onto the space $\mathbf{p}^{\perp}$. Then, $y(t)$ can be extracted as $y(t)=\mathbf{p}^{T} \mathbf{x}(t)$ in which the scalar $\frac{1}{\mathbf{p}^{T} \mathbf{q}}$ has been omitted and $\mathbf{q}$ has been dropped from both sides of the equation. In second-order statistics based BSE [11], both $\mathbf{p}$ and $\mathbf{q}$ are unknown and in order to extract one source the following cost function has been proposed

$$
[\hat{\mathbf{d}}, \hat{\mathbf{p}}, \hat{\mathbf{q}}]=\arg \min _{\mathbf{d}, \mathbf{p}, \mathbf{q}} J_{M}(\mathbf{d}, \mathbf{p}, \mathbf{q})
$$

where $J_{M}(\mathbf{d}, \mathbf{p}, \mathbf{q})=\sum_{k=1}^{K}\left\|\mathbf{R}_{k} \mathbf{p}-d_{k} \mathbf{q}\right\|_{2}^{2}, \mathbf{d}$ is a column vector $\mathbf{d}=\left[d_{1}, d_{2}, \cdots, d_{K}\right]^{T}$ and $\|\cdot\|_{2}^{2}$ denotes the squared Euclidean norm. The cost function in (2) utilizes the fact that for BSE, $\mathbf{R}_{k} \mathbf{p}$ should be collinear with $\mathbf{q}$ incorporating the coefficients $d_{k}$ which provides $\mathbf{q}$ with the proper scaling. The trivial answer for (2) is $\mathbf{d}=\mathbf{p}=\mathbf{q}=\mathbf{0}$. This solution has been avoided by imposing the condition $\|\mathbf{q}\|_{2}=\|\mathbf{d}\|_{2}=1$. Successful minimization of (2) leads to the identification of $\mathbf{p}$ which extracts $y(t)$, the source of interest (SOI).

However, it is not possible to determine which source is extracted. Therefore, some prior knowledge should be incorporated into the separation process to extract only the SoI. To this end, we consider an auxiliary cost function $J_{A u x}=$ $\sum_{k=1}^{K}\left\|b_{k} \mathbf{q}-\boldsymbol{\mu}_{\mathbf{q}}\right\|_{2}^{2}$ where $\boldsymbol{\mu}_{\mathbf{q}}$ is the estimation of $\mathbf{q}$ provided by PARAFAC (see section 2.2) and $\mathbf{b}$ is a column vector $\mathbf{b}=\left[b_{1}, b_{2}, \cdots, b_{K}\right]^{T}$. By minimizing $J_{A u x}$ coupled with (2) in a Lagrangian framework, i.e., $J_{t o t}=J_{M}+\eta_{\mathbf{q}} J_{A u x}$, we effectively extract the SoI as the first extracted source. In other words, the new cost function becomes

$$
[\hat{\mathbf{b}}, \hat{\mathbf{d}}, \hat{\mathbf{p}}, \hat{\mathbf{q}}]=\arg \min _{\mathbf{b}, \mathbf{d}, \mathbf{p}, \mathbf{q}} \sum_{k=1}^{K}\left(\left\|\mathbf{R}_{k} \mathbf{p}-d_{k} \mathbf{q}\right\|_{2}^{2}+\eta_{\mathbf{q}}\left\|b_{k} \mathbf{q}-\boldsymbol{\mu}_{\mathbf{q}}\right\|_{2}^{2}\right)
$$

where $\eta_{\mathbf{q}}$ is the Lagrange multiplier. In (3), the $b_{k}, k=$ $1,2, \cdots, K$ values are free parameters to scale $\mathbf{q}$ during an iterative solution to (3) and $\|\mathbf{b}\|_{2}=1$.

The solution to (3) is found by alternatively adjusting its parameters. Firstly, we fix $\mathbf{q}, \mathbf{d}$, and $\mathbf{b}$ and update $\mathbf{p}$. Taking the gradient of $J_{t o t}$ with respect to $\mathbf{p}$ leads to an optimal analytical solution for $\mathbf{p}$ as $\frac{\partial J_{t o t}}{\partial \mathbf{p}}=2 \sum_{k=1}^{K} \mathbf{R}_{k}\left(\mathbf{R}_{k} \mathbf{p}-d_{k} \mathbf{q}\right)=$ $\mathbf{0}$ and

$$
\mathbf{p} \Leftarrow \mathbf{Q}\left(\sum_{k=1}^{K} d_{k} \mathbf{R}_{k}\right) \mathbf{q} ; \mathbf{Q}=\left[\sum_{k=1}^{K}\left(\mathbf{R}_{k}\right)^{2}\right]^{-1}
$$

where $a \Leftarrow b$ denotes replacing $a$ by $b$. Thereafter, we fix $\mathbf{p}, \mathbf{b}$, and $\mathbf{q}$ and update $\mathbf{d}$. As in [11], utilizing the property that $\|\mathbf{q}\|_{2}=1$, the gradient of $J_{\text {tot }}$ with respect to $d_{k}$ becomes $\frac{\partial J_{\text {tot }}}{\partial d_{k}}=-2 \sum_{k=1}^{K}\left(\left(\mathbf{R}_{k} \mathbf{p}\right)^{T}-d_{k} \mathbf{q}^{T}\right) \mathbf{q}=0, k=$ $1,2, \cdots, K$. The update rule for $\mathbf{d}$ is as

$$
\mathbf{d} \Leftarrow \frac{\mathbf{u}}{\|\mathbf{u}\|_{2}} ; \mathbf{u}=\left[\mathbf{r}_{1}^{T} \mathbf{q}, \mathbf{r}_{2}^{T} \mathbf{q}, \cdots, \mathbf{r}_{k}^{T} \mathbf{q}\right]^{T} .
$$

where $\mathbf{r}_{k}=\mathbf{R}_{k} \mathbf{p}$. Then, fixing $\mathbf{p}, \mathbf{d}$, and $\mathbf{b}$, we adjust $\mathbf{q}$ while ensuring $\|\mathbf{q}\|_{2}=1$. Considering $\frac{\partial J_{t o t}}{\partial \mathbf{q}}=-2 \sum_{k=1}^{K} d_{k} \mathbf{r}_{k}-$ $2 \eta_{\mathbf{q}} \sum_{k=1}^{K} b_{k} \boldsymbol{\mu}_{\mathbf{q}}+2(1+\eta) \mathbf{q}=\mathbf{0}, \mathbf{q}$ is adjustable by

$$
\mathbf{q} \Leftarrow \frac{\mathbf{v}}{\|\mathbf{v}\|_{2}} ; \mathbf{v}=\sum_{k=1}^{K}\left(d_{k} \mathbf{r}_{k}+\frac{1}{K} \eta_{\mathbf{q}} b_{k} \boldsymbol{\mu}_{\mathbf{q}}\right) .
$$

For updating $\mathbf{b}$, the rest of the variables are fixed, i.e., $\mathbf{q}, \mathbf{p}$, and $\mathbf{d}$ and we proceed by minimizing (3) with respect to $b_{k}$, i.e., $\frac{\partial J_{t o t}}{\partial b_{k}}=2 \eta_{\mathbf{q}} \sum_{k=1}^{K}\left(b_{k}-\boldsymbol{\mu}_{\mathbf{q}}^{T} \mathbf{q}\right)=0$. $\mathbf{b}$ is updated as

$$
\mathbf{b} \Leftarrow \frac{\mathbf{w}}{\|\mathbf{w}\|_{2}} ; \mathbf{w}=\left[\boldsymbol{\mu}_{\mathbf{q}}^{T} \mathbf{q}, \boldsymbol{\mu}_{\mathbf{q}}^{T} \mathbf{q}, \cdots, \boldsymbol{\mu}_{\mathbf{q}}^{T} \mathbf{q}\right] .
$$

We retain $\mathbf{b}$ as a vector instead of a scalar to present consistent formulation.

Finally, in order to solve (3) for the Lagrange multiplier we define vector $\mathbf{e}_{i}$ as a vector whose elements are all zero except for the $i$ th which is one, i.e., $\mathbf{e}_{i}=[0, \cdots, 0,1,0, \cdots, 0]^{T}$, $\forall i \in\{1,2, \cdots, K\}$. From (6), in each iteration we assign a new value for $\eta_{\mathbf{q}}$ as

$$
\eta_{\mathbf{q}}=\frac{\left[\frac{1}{b_{i}}\left(\mathbf{v}-\sum_{k=1}^{K} d_{k} \mathbf{r}_{k}\right)\right]^{T} \mathbf{e}_{i}}{\boldsymbol{\mu}_{\mathbf{q}}^{T} \mathbf{e}_{i}} .
$$

Through consistent simulation studies, we have found consistent convergence of this optimization scheme as reported in Section 3. Next, we establish how PARAFAC is utilized to provide the required a priori information. 


\subsection{PARAFAC}

PARAFAC is a widely accepted tool in extracting disjoint multi-dimensional phenomena $[9,10,12]$. In this paper, by exploiting PARAFAC, we extract the factor relevant to the eye-blink artifact for use within the SBSE. The resulting spatial signature of the eye-blink-related factor, i.e., $\boldsymbol{\mu}_{\mathbf{q}}$ is exploited to formulate (3). In our approach, the multi-channel EEG data are transformed into the time-frequency domain. This gives the two-way EEG recording, i.e., the matrix of space(channel)-time with an extra dimension, i.e., frequency and yields a three-way array of space-time-frequency. In other words, for $I$ EEG channels, we compute the energy of the time-frequency transform for $J$ time instants and $K$ frequency bins. By stacking these $I$ matrices (of size $J \times K$ ) and adopting the MATLAB matrix notation we set up the three-way array as $\mathbf{X}^{I \times J \times K} \equiv \mathbf{X}(1: I ; 1: J ; 1: K)$ and introduce it to PARAFAC. To setup a three-way array, in the present study, we follow the same procedure as in [10] where the continuous wavelet transform is utilized to provide a time-varying representation of the energy of the signal over all channels. The complex Morlet's wavelets $w\left(t, f_{0}\right)$, with $\sigma_{f}=1 /\left(2 \pi \sigma_{t}\right)$, and $A=\left(\sigma_{t} \sqrt{\pi}\right)^{-1 / 2}$, is used here in which the trade-off ratio $\left(f_{0} / \sigma_{f}\right)$ is 7 , to create a wavelet family. This wavelet configuration is known to be optimized in EEG processing [10].

In mathematical terms, the factor analysis is expressed as $\mathbf{X}^{I \times J}=\mathbf{A}^{I \times F}\left(\mathbf{S}^{J \times F}\right)^{T}+\mathbf{E}^{I \times J}$ where $\mathbf{A}$ is the factor loading, $\mathbf{S}$ the factor score, $\mathbf{E}$ the error, and $F$ is the number of factors. Similarly, the PARAFAC for the three-way arrays $\mathbf{X}^{I \times J \times K}$ is presented by unfolding one modality to another as $\mathbf{X}^{I \times J K}=\mathbf{A}^{I \times F}\left(\mathbf{S}^{K \times F} \odot \mathbf{D}^{J \times F}\right)^{T}+\mathbf{E}^{I \times J K}$ where $\mathbf{D}$ is the factor score corresponding to the second modality and $\odot$ denotes the Khatri-Rao product [9]. Equivalently, the $j$ th matrix corresponding to the $j$ th slice of the second modality of the 3-way array is expressed as

$$
\mathbf{X}^{I \times j \times K}=\mathbf{A}^{I \times F} \mathbf{D}_{j}^{F \times F}\left(\mathbf{S}^{K \times F}\right)^{T}+\mathbf{E}^{I \times j \times K}
$$

where $\mathbf{D}_{j}$ is a diagonal matrix having the $j$ th row of $\mathbf{D}$ along the diagonal. The method of alternative least square (ALS) is the most common way to estimate the PARAFAC model. In order to decompose the multi-way array to parallel factors the cost function (normally the squared error) is minimized as [9]

$$
[\hat{\mathbf{A}}, \hat{\mathbf{S}}, \hat{\mathbf{D}}]=\arg \min _{\mathbf{A}, \mathbf{S}, \mathbf{D}}\left\|\mathbf{X}^{I \times J K}-\mathbf{A}^{I \times F}\left(\mathbf{S}^{K \times F} \odot \mathbf{D}^{J \times F}\right)^{T}\right\|_{2}^{2}
$$

Here, $\mathbf{X}^{I \times J \times K}$ is the three-way array of wavelet energy of multi-channel EEG recordings and $\mathbf{A}^{I \times F}, \mathbf{S}^{K \times F}$, and $\mathbf{D}^{J \times F}$ respectively denote the spatial, temporal, and spectral signatures of $\mathbf{X}^{I \times J \times K}$. For details of the uniqueness of PARAFAC model in cases of real and complex multi-way arrays the interested reader is referred to [12].

\section{RESULTS}

We applied the proposed algorithm to real EEG measurements. The database was provided by King's College Hospital, London, UK, and represent a wide range of patients and, therefore, give a proper evaluation of our method. The scalp EEG was obtained using 16 Silver/Silver-Chloride electrodes placed at locations defined by the 10-20 system [1]. The data were acquired using a Beekeeper Telefactor EEG amplifier, sampled at $200 \mathrm{~Hz}$, and bandpass filtered with cut-off frequencies of $0.3 \mathrm{~Hz}$ and $40 \mathrm{~Hz}$. Each EEG segment was transformed into the time-frequency domain by means of complex wavelet transform where the frequency band of from $2 \mathrm{~Hz}$ to $20 \mathrm{~Hz}$ with resolution of $0.1 \mathrm{~Hz}$ has been considered. This threeway array is then introduced to PARAFAC where the number of factors is selected as one or two, as highlighted in the following experiments, identified by using the method of core consistency diagnostic (CORCONDIA) [9].

The left subplot of Fig. 1 shows EEG measurements contaminated by two eye-blinks at approximate times of two and half and five seconds. The effects of the eye-blinks are evident mostly in the frontal electrodes, namely, FP1, FP2, F3, F4, F7, and F8. However, central, i.e., C3 and C4 and occipital, i.e., O1 electrodes are also affected. Implementation of the PARAFAC on this measurement results in the STF model, i.e., the spectral, temporal, and spatial signatures which are depicted in Fig. 2-(a to c). Although there are two eye-blinks CORCONDIA suggests the number of factors $F$ to be as in one Fig. 2-(d). This value is rational since both of the eyeblinks originate from a certain vicinity (frontal lobe of the brain) and occupy the same frequency band and there is no significant brain background activity.

In order to minimize (3) the initial values of the vectors $\mathbf{b}, \mathbf{d}, \mathbf{p}$, and $\mathbf{q}$ are independently drawn from standardized normal distributions, i.e., $N(0,1)$ and $\boldsymbol{\mu}_{\mathbf{q}}$ is set to the spatial signature of the extracted factor. Fig. 3 compares the average value of the $10 \log _{10} \frac{J_{t o t}}{N K}$ over 50 independent experiments. Two scenarios have been devised by varying the number of time lags, i.e., $K=10$ and 25 . Note that in [11] $J_{\text {tot }}=J_{M}$. Evidently, in both scenarios the performance of the proposed SBSE method is superior to that of the method [11]. Usually, after approximately 10 iterations the extracting vector, i.e., p, is identified. Furthermore, by incorporating the priori knowledge, it is guaranteed that $\mathbf{p}$ extracts the eye-blink source. The effect of the eye-blink is then removed from the multi-channel EEG using the batch deflation algorithm in [2]. Extensive simulations demonstrate that the proposed methodology can efficiently identify and remove the eye-blink artifact from raw EEG measurements.

\section{CONCLUSION}

We have presented a robust method for removing EOG from EEG recordings by providing the BSE algorithm with the es- 

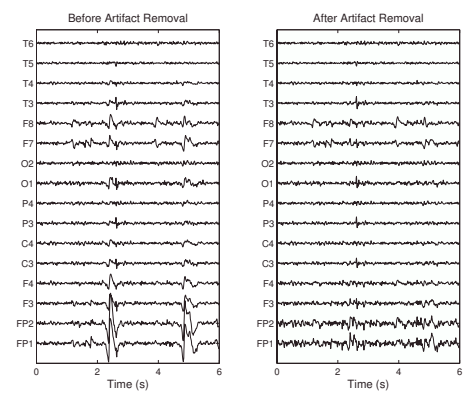

Fig. 1. The result of the proposed eye-blink artifact removal method applied to a set of real EEGs.

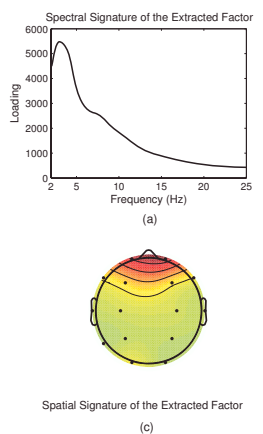

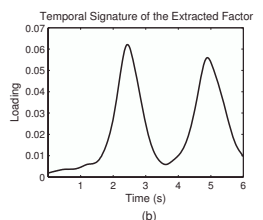

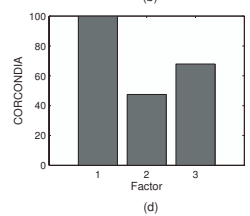

Fig. 2. The extracted factor by using PARAFAC; (a) and (b) illustrate respectively the spectral and temporal signatures of the extracted factor and (c) represents the spatial distribution of the extracted factor which has been utilized during extraction procedure.

timation of the column of the mixing matrix corresponding to the eye-blink source. The vector of spatial distribution of the eye-blink factor has been identified using PARAFAC. The EEGs are processed using the time-lagged second-order BSE algorithm and the artifact is autonomously cancelled; then, the EEGs are reconstructed in a deflation framework. Based on our experiments, the proposed SBSE algorithm consistently removes the eye-blink artifacts from the EEG signals.

\section{REFERENCES}

[1] S. Sanei and J. A. Chambers, EEG Signal Processing, Wiley, New York, 2007.

[2] A. Cichocki and S. Amari, Adaptive Blind Signal and Image Processing-Learning Algorithms and Applications, Wiley, New York, 2005.

[3] M. Fatourechi, A. Bashashati, R. K. Ward, and G. E. Birch, "EMG and EOG artifacts in brain computer interface systems: A survey," Clin. Neurophysiol., 2007, in Press.

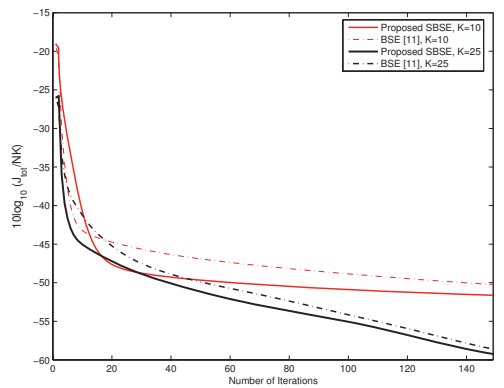

Fig. 3. The averaged (over 50 independent runs) convergence characteristics, $10 \log _{10} \frac{J_{t o t}}{N K}$, of the SBSE and BSE of [11] are depicted for two values of $K$, i.e., 10 and 25 .

[4] A. Schlögl, C. Keinrath, D. Zimmermann, R. Scherer, R. Leeb, and G. Pfurtscheller, "A fully automated correction method of EOG artifacts in EEG recordings," Clin. Neurophysiol., vol. 118, pp. 98-104, 2007.

[5] K. H. Ting, P. C. W. Fung, C. Q. Chang, and F. H. Y. Chan, "Automatic correction of artifact from single-trial event-related potentials by blind source separation using second order statistics only," Med. Eng. Phys., vol. 28, pp. 780-794, 2006.

[6] L. Shoker, S. Sanei, and J. A. Chambers, "Artifact removal from electroencephalograms using a hybrid BSSSVM algorithm," IEEE Sig. Process. Lett., vol. 12, no. 10, pp. 721-724, 2005.

[7] C. J. James and O. J. Gibson, "Temporally constrained ICA: An application to artifact rejection in electromagnetic brain signal analysis," IEEE Trans. Biomed. Eng., vol. 50, no. 9, pp. 1108-1116, 2003.

[8] A. C. Soong and P. Z. Koles, "Principal-component localization of sources of the background EEG," IEEE Trans. Biomed. Eng., vol. 42, no. 1, pp. 59-67, 1995.

[9] R. Bro, "PARAFAC: Tutorial and applications," Chemometrics Intell. Lab. Syst., vol. 38, pp. 149-171, 1997.

[10] F. Miwakeichi, E. Martinez-Montes, P. A. Valdés-Sosa, N. Nishiyama, H. Mizuhara, and Y. Yamaguchia, "Decomposing EEG data into space-time-frequency components using parallel factor analysis," NeuroImage, vol. 22, pp. 1035-1045, 2004.

[11] X. Li and X. Zhang, "Sequential blind extraction adopting second-order statistics," IEEE Sig. Process. Lett., vol. 14 , no. 1 , pp. 58-61, 2007.

[12] N. D. Sidiropoulos, G. B. Giannakis, and R. Bro, "Blind PARAFAC receivers for DS-CDMA systems," IEEE Trans. Sig. Process., vol. 48, pp. 810-823, 2000. 